\title{
TRANSMUTACIÓN TEXTO-ILUSTRACIÓN: UN MODELO PARA EL ANÁLISIS DE SU EFECTO SOBRE LAS DINÁMICAS Y FUNCIONES DE LOS LIBROS INFANTILES
}

\author{
Text-Illustration Transmutation: A Model for the Analysis of its Effect \\ ON THE Dynamics AND Functions OF Children's BoOKS
}

TRANSMUTATIONS TEXTE-ILLUSTRATION DANS DES LIVRES POUR ENFANTS : UN MODÈLE POUR ANALYSER LEUR EFFET SUR LA DYNAMIQUE ET LE FONCTIONNEMENT DES LIVRES POUR ENFANTS

\section{Bruno Echauri-Galván}

M. A. Investigación en Literaturas

Anglófonas e Hispánicas Contemporáneas, Universidad de Alcalá.

Profesor ayudante doctor, Departamento de Filología Moderna, Universidad de Alcalá, y miembro de los grupos de investigación RECEPTION y en Literatura Contemporánea de la Universidad de Alcalá. Calle Trinidad, 3, 28801, Alcalá de Henares, Madrid, España. bruno.echauri@uah.es https://orcid. org/0000-0002-7055-5699

\section{RESUMEN}

Este artículo busca aplicar ciertos fundamentos de la teoría de la traducción al análisis de álbumes ilustrados. Con este fin, presenta un modelo de análisis para el estudio de las transmutaciones entre texto e ilustración en los libros infantiles, dividido en tres etapas. En la primera, se determina la información que el elemento visual (dibujo) traslada, añade, modifica u omite con respecto al elemento verbal (texto), a partir de la identificación de distintas estrategias y procedimientos propios de la traducción. En la segunda etapa, se aplican estos resultados al estudio de las dinámicas entre texto e ilustración, buscando establecer qué mensaje acaba transmitiendo al lector la confluencia entre estos dos elementos. En la tercera, se identifican varias de las funciones tradicionalmente asociadas a la literatura infantil, para calibrar hasta qué punto la interrelación entre el texto y la ilustración las enfatiza o eclipsa. Para ejemplificar el modelo de análisis anterior se estudian distintos pasajes e ilustraciones del libro de Marla Frazee, A Couple of Boys Have the Best Week Ever.

Palabras clave: álbum ilustrado; dinámicas texto-ilustración; literatura infantil; teoría de la traducción.

\begin{abstract}
The present paper aims to apply theoretical aspects of translation studies to the analysis of picture books. For this purpose, it introduces a three-stage analytical model aimed at the study of text-picture transmutations in the aforementioned genre. In the first stage, the information that the visual element (picture) conveys, adds, omits or modifies compared to the verbal element (text) is identified using different translation strategies and procedures. In a second stage, these results are used for the study of text-picture dynamics, which determine the message that both elements' convergence will eventually transmit. In the third one, the information that stems from the previous stages is used to identify some of the main functions of children's literature and to establish to which extent they are foregrounded or
\end{abstract}

Received: 2018-08-04 / Accepted: 2019-07-10 / Published: 2020-01-28

http://doi.org/10.17533/udea.ikala.v25n01a08 
overshadowed by the interrelationship between texts and pictures. As a means to exemplify the application of this analytical model, the paper studies various passages and their corresponding illustrations from Marla Frazee's $A$ Couple of Boys Have the Best Week Ever.

Keywords: picture books; text-picture dynamics; children's literature; translation theory.

\section{RÉSUMÉ}

Dans cet article, on cherche à appliquer certains fondements de la théorie de la traduction à l'analyse des livres illustrés. Il présente un modèle d'analyse en trois étapes pour étudier les transmutations texte-illustration dans les livres pour enfants. Premièrement, des stratégies et procédés typiques de la traduction sont employés pour déterminer l'information transmise, ajoutée, modifiée ou omise par l'élément visuel (dessin) par rapport à l'élément verbal (texte). Ces résultats sont ensuite utilisés dans l'étude des dynamiques texte-illustration, qui servent à déterminer le message reçu par le lecteur grâce à ces deux éléments. Finalement, cela permet d'identifier plusieurs des fonctions traditionnellement associées à la littérature pour enfants et d'évaluer dans quelle mesure l'interaction entre texte et illustration est masquée ou, par contre, mise en valeur par ces fonctions. Pour illustrer ce modèle d'analyse, nous étudions divers passages et illustrations du livre A Couple of Boys Have the Best Week Ever écrit par Marla Frazee.

Mots clés : album ilustré ; dynamiques texte-illustration ; littérature pour enfants ; théorie de la traduction. 


\section{Introducción}

Existe poco consenso en torno a qué se considera exactamente "literatura infantil". Por un lado, resulta muy complicado determinar qué es un texto dirigido a un público infantil y, de existir esta categoría, cómo deberían ser los libros que en ella se incluyen (Hunt, 2001). Por otro, varios ejemplos a lo largo de la historia de la literatura (Las aventuras de Tom Sawyer de Mark Twain, muchos cuentos de Rudyard Kipling y tantos otros casos) han demostrado que las fronteras que tratan de poner coto a estos textos son, en muchas ocasiones, móviles y difusas, ya sea por su temática, sus múltiples lecturas, el tono, la ambivalencia del autor $\mathrm{u}$ otros factores igualmente relevantes (Hunt, 2001).

En esta larga y compleja discusión se encuadra un género tradicionalmente asociado a los niños por su aparente simpleza, pero que presenta las mismas aristas y problemáticas a la hora de ser etiquetado como "infantil": el álbum ilustrado. También conocido como "libro-álbum", se trata de un género de alto contenido visual, en el que texto e imagen se combinan a lo largo de la obra y donde ambos elementos interactúan en la creación de significado.

El libro en el que se centra el análisis de este artículo se enmarca en esta categoría. A Couple of Boys Have the Best Week Ever es una historia escrita e ilustrada por la autora americana Marla Frazee (2008), reconocida figura dentro del género. En este trabajo narra las andanzas de dos niños, Eamon y James, durante una semana de verano en casa de los abuelos del primero, en la que acuden a un campamento en plena naturaleza. Allí vivirán nuevas y emocionantes experiencias, y muy variadas aventuras. Dirigido, en principio, a niños de entre seis y nueve años, el libro destaca por sus numerosos toques de humor e ironía, expresados muchas veces a través de la combinación entre palabras e imágenes, característica del álbum ilustrado.

Esta relación entre elementos verbales y visuales es el punto de partida del presente trabajo, que se apoya en el concepto seminal de traducción intersemiótica (o transmutación) acuñado por Jakobson
(1959/2000), y en otros posteriores como el de recreación (Pereira, 2008), para defender las coincidencias existentes entre los procesos de traducción e ilustración. Trabajar sobre esta asunción permite crear un modelo de análisis dividido en tres etapas y orientado a explorar las transmutaciones texto-ilustración a través de estrategias y procedimientos propios de los estudios traductológicos. Partiendo de estas bases, se pretende explorar dos hipótesis de partida:

1. El estudio de las recreaciones texto-ilustración por medio de estrategias y procedimientos de traducción permite identificar las dinámicas texto-imagen de una forma precisa, ya que posibilita subrayar la información que se mantiene, omite, añade o modifica en el elemento verbal y el visual.

2. Dependiendo de los procedimientos traductológicos utilizados en la conformación de la imagen y de las dinámicas creadas entre ambos elementos a partir de ellos, se enfatizan o difuminan ciertas funciones de la literatura infantil.

Para ello, se lleva a cabo un análisis de tres fragmentos (y sus correspondientes ilustraciones) del libro de Frazee (2008) siguiendo el modelo antes mencionado. Su implementación implica, en primer lugar, determinar las estrategias y los procedimientos de traducción presentes en la elaboración de la imagen; posteriormente, se identifican las dinámicas texto-ilustración que de ellos se derivan; por último, se presentan algunas de las funciones asociadas tradicionalmente a la literatura infantil que estas dinámicas pueden potenciar u opacar en la obra en cuestión.

\section{Marco teórico}

Como se mencionaba en la sección anterior, una de las nociones centrales alrededor de las que pivota este artículo es la de traducción intersemiótica o transmutación, la cual, según Jakobson (1959/2000), se produce entre un código de signos verbales y otro de signos no verbales. Este tipo de traducción 
puede catalogarse como autónoma, caso de una novela hecha película, o como complementaria, caso de una foto que acompaña a un artículo en el periódico. A partir de estos conceptos, el paso de los años ha visto cómo la idea original de este autor se examinaba y ramificaba hasta cristalizar en distintas teorías que aluden a las traducciones entre códigos semióticos diferentes.

Una de las más destacadas es, sin duda, la desarrollada por Eco (2008), quien revisita los tres tipos de traducción propuestos por Jakobson, para establecer una clara distinción entre traducir e interpretar. Para Eco (2008), una traducción siempre es, en cierto grado, una interpretación, concepción que no se aplica en sentido inverso. Y es que, según este autor, para que exista traducción tiene que haber, indefectiblemente, una interpretación previa del texto que la desencadene. Toda traducción que no cumpla este presupuesto será, en su opinión, una traducción de baja calidad. En lo que concierne a los procesos de transmutación, Eco (2008) defiende que, en casos como estos en los que se produce cambio de materia, resulta extremadamente complejo trazar equivalencias específicas entre la obra fuente y la obra meta, por lo que no deberíamos hablar de "operaciones traductológicas" sino, más bien, de "operaciones interpretativas”. Así, desliga muchos ejemplos de transmutación de la idea tradicional de "traducción".

Estando fundamentalmente de acuerdo con el hecho de que traducir una obra implica, en cierta medida, un proceso de interpretación, considero que los casos sobre los que se construye este trabajo (i. e., transmutaciones texto-ilustración) no deberían desgajarse por completo de la disciplina de la traducción y los conceptos que la integran. $\mathrm{Y}$ es que, ya sea de manera consciente o inconsciente, el ilustrador tiende a emplear metodologías similares a las del traductor a la hora de convertir en dibujo un pasaje concreto de un texto (Pereira, 2008), lo cual permite trazar conexiones a nivel traslativo entre la obra original y la imagen resultante.

Por esta razón, este artículo entronca con la idea de Pereira (2008) de que toda traducción (in- cluidas aquellas de carácter intersemiótico) de una obra artística es, al fin y al cabo, una "recreación" del original. Aplicado a las transmutaciones textoilustración, este concepto defiende que, a pesar de la labor interpretativa que inherentemente acompaña al arte de ilustrar, el producto final sigue siendo un resultado que deriva del texto fuente y, por tanto, analizable en términos traductológicos.

En esta línea y en relación con la obra en la que se centra el análisis de este artículo, es relevante subrayar que tanto el texto como las ilustraciones son obra de la misma autora. Puesto que Frazee (2008) es quien escribe e ilustra las páginas de este libroálbum, es incluso más pertinente que en otros casos hablar de una "recreación de la palabra en la imagen" y no de una "nueva interpretación", ya que las ideas e impulsos surgen, en ambos planos, de la misma fuente.

\section{Estrategias y procedimientos de traducción}

Como se anunciaba en el apartado anterior, este artículo se centra en las interrelaciones existentes entre la traducción y la ilustración de libros, área que ya ha sido explorada previamente por autores como Sitzia (2010) o Salmani y Eghtesadi (2015), entre otros. Estos trabajos han abordado planos muy diferentes del proceso de transmutación entre textos e ilustraciones. Sitzia (2010), por ejemplo, aplica la noción de transmutación a una edición de Cendrillon (Cenicienta) del siglo XIX, ilustrada por Gustave Doré, centrando su análisis en los aportes de información de las ilustraciones al texto y en las implicaciones que los mensajes contenidos en el elemento visual tienen (tuvieron) sobre el público receptor. Por otro lado, el trabajo de Salmani y Eghtesadi (2015) emplea el concepto de transmutación en el estudio de la evolución de las portadas de distintos libros. Para ello, utiliza distintos parámetros de análisis, como la ideología, la información lingüística exhibida, el modo de representación o la parte de la historia que refleja cada portada en cuestión.

El caso que nos ocupa, sin embargo, plantea un enfoque distinto a los trabajos descritos 
anteriormente y prosigue el camino marcado por Pereira (2007) en su análisis de las transmutaciones entre texto e ilustración en Las aventuras de Alicia en el País de las Maravillas, en el que ya aplicó procedimientos de traducción como la omisión, la condensación o la traducción literal al estudio de relatos ilustrados. El autor buscaba así subrayar los paralelismos existentes entre las decisiones y metodologías que, en tantas ocasiones, traductores e ilustradores toman y utilizan. El presente artículo pretende ampliar estas bases y reforzar las analogías entre traducción e ilustración al incluir nuevos procedimientos, sumar las estrategias de traducción al estudio de las transmutaciones entre texto y dibujo, y emplear ambas herramientas no solo en el análisis de la obra original y meta, sino también de las sinergias que se crean entre ellas.

Sin embargo, a pesar de lo expuesto hasta ahora, conviene señalar que las diferencias entre la traducción propiamente dicha y la ilustración de un texto, por supuesto, existen. A las inevitables variaciones en la realización estética que se producen en la traducción de cualquier obra artística (Campos, 1992), hay que añadir las lógicas divergencias derivadas del cambio de materia y de la comparación entre dos disciplinas, escritura e ilustración, que componen sus obras con instrumentos distintos. Al respecto, el paso a un sistema de signos no lingüísticos que los procesos de transmutación explorados en este artículo implican, presupone una lógica redefinición conceptual de algunas de las estrategias y los procedimientos traductológicos empleados, ya que una aplicación igual a la que se realiza con traducciones interlingüísticas resulta, en ocasiones, difícil de implementar.

Así, en los ejemplos analizados, se utilizan los siguientes conceptos traductológicos: a nivel de estrategias (esto es, el enfoque general que el traductor da a su texto meta), se distingue entre traducción directa y oblicua (Vinay y Darbelnet, 1995). Grosso modo y originalmente, la primera se entiende como una traducción literal, mientras que la segunda viene ligada al concepto de traducción libre. Para los propósitos de este artículo, significados muy similares resultan aplicables: una estrategia directa busca que las ilustraciones reflejen, en la medida de lo posible, una información igual o muy próxima a la expresada en el texto, mientras que una traducción oblicua sería un caso de traducción libre en la que el ilustrador decide modificar ciertas partes de la fuente original, de modo que el mensaje resultante varíe significativa o muy significativamente con respecto al elemento verbal. Llegados a este punto, conviene subrayar que ambos enfoques no son necesariamente excluyentes, sino que pueden coexistir a lo largo de una misma obra meta.

Por otro lado, cuando hablamos de procedimientos de traducción, lo hacemos de decisiones tomadas por el traductor sobre unidades concretas del texto. $\mathrm{Si}$ bien existen numerosas categorizaciones al respecto, los identificados a lo largo del análisis pertenecen al listado elaborado por Hurtado Albir (2001). La relación posterior aporta su acepción más común y, cuando corresponda, la que se aplica en el apartado de análisis:

- Traducción literal: en contextos interlingüísticos, traducción palabra por palabra. En el caso de la transmutación texto-ilustración, este procedimiento implica plasmar en la ilustración (con las modificaciones propias del cambio entre un sistema verbal y uno no verbal) segmentos de información iguales a los contenidos en el texto.

- Amplificación: en su definición original, este procedimiento implica el añadido de elementos no mencionados en el texto fuente. En este caso, la idea inicial es perfectamente aplicable al análisis de los pasajes e ilustraciones del libro de Frazee (2008), por lo que se considerarán amplificaciones aquellos elementos añadidos al dibujo que el texto no recoge. Un procedimiento similar también aplicable a las transmutaciones texto-ilustración es el de explicitación. La principal diferencia con la amplificación, en este caso, es que los elementos añadidos en el dibujo pueden deducirse de lo expresado en el elemento textual, algo que no ocurre con las amplificaciones. Sin embargo, como se verá en los ejemplos objeto de análisis, no 
hay ningún caso de explicitación en los elementos estudiados.

- Elisión: este procedimiento es contrario a la amplificación e implica la omisión de elementos presentes en el texto original. Como en el caso anterior, la definición original del procedimiento resulta aplicable a nuestro modelo de análisis, por lo que se identificarán como elisiones los elementos textuales que Frazee decide omitir en sus dibujos.

- Variación: consiste en la modificación de elementos lingüísticos o paralingüísticos que afectan a distintos aspectos, como el registro, el estilo o el tono del texto meta. En el marco de este artículo, este procedimiento tiende a ser consecuencia de otros que hacen que el tono, el mensaje o el contexto final creado por la autora a través del elemento visual, varíe en relación con lo explicitado en el texto.

Completar un análisis de este tipo sobre transmutaciones texto-ilustración permite identificar y etiquetar las decisiones del ilustrador y compararlas con el texto fuente a un nivel similar al de un análisis comparativo entre un texto original y su traducción a otra lengua. Pero, además, como veremos posteriormente, los resultados obtenidos en este caso pueden emplearse también para sustentar el estudio de otros elementos propios de los álbumes ilustrados.

\section{Dinámicas texto-ilustración}

Una de las principales particularidades del proceso de transmutación que se analiza en este artículo es que la fuente original y la obra meta operan a la vez y, en muchas ocasiones, incluso en la misma página. En otras palabras, el elemento visual resultante no se interpreta en el vacío, sino en conjunción con el texto del que deriva. Esta interacción entre las partes puede generar distintas dinámicas extensamente estudiadas y clasificadas. Pese a la existencia de otras taxonomías y otros términos como los propuestos por Schwarcz (1982) o Sipe (1998), en este artículo se emplea la propuesta de Nikolajeva y Scott (2001) y su clasificación de las potenciales dinámicas entre texto e ilustración.

Según estas autoras, dichas dinámicas pueden catalogarse como simétricas, de ampliación, complementarias, de contrapunto o contradictorias. Dentro de estas cinco categorías, la simetría hace referencia a los casos en los que el texto y la ilustración reproducen la misma información, esto es, "cuentan la misma historia”.

La ampliación, por su parte, supone que la ilustración aporta nueva (aunque muy limitada) información con respecto al texto o viceversa.

Cuando esto ocurre a un nivel más significativo y uno de los dos elementos aporta información verdaderamente relevante y diferente al otro, nos encontramos ante un caso de complementariedad.

En las dinámicas de contrapunto, el texto y la imagen se conjugan para transmitir significados que van más allá del ámbito individual de cada uno, pudiendo llegar a aportar distintos ángulos a la trama.

Casos extremos de contrapunto derivan en dinámicas contradictorias, que engloban aquellos ejemplos en los que el elemento verbal y el elemento visual ofrecen informaciones opuestas y, de algún modo, versiones contrarias de la historia.

Si ponemos el foco sobre las definiciones propuestas para cada categoría, podremos observar que toda dinámica puede considerarse, al fin y al cabo, consecuencia de distintas acciones traslativas, ya que depende de la cantidad y la relevancia de la información que se replica, añade, omite, modifica o transforma en el elemento visual a partir del texto. Un análisis de la relación entre el texto y la imagen mediante las estrategias y los procedimientos traductológicos presentados en el apartado anterior (y otros que no se observan en los ejemplos analizados en este trabajo) permite, en primera instancia, subrayar los fenómenos ocurridos en el trasvase de información para, posteriormente, determinar su 
grado de trascendencia en la conformación de la dinámica y, por consiguiente, en el mensaje que acabará transmitiendo el binomio texto-ilustración. En este sentido y como se puede comprobar en el análisis, ciertas amplificaciones pueden, por ejemplo, cambiar el tipo de dinámica texto-ilustración en función de la información que aporten. En otros casos, el mismo procedimiento puede no tener un impacto significativo sobre la dinámica.

El modelo de análisis propuesto en este artículo busca, por tanto, explorar en profundidad el tipo de dinámica que se da en cada caso, conectando este concepto con los estudios de traducción. Esta disciplina aporta herramientas concretas que incrementan la precisión del análisis (permitiendo identificar y distinguir, por ejemplo, aquella información que se explicita de la que directamente se añade por amplificación) y que, por consiguiente, refuerzan la solidez de la discusión que se genera a raíz de cada ejemplo.

Además, dado el papel que las dinámicas texto-ilustración pueden cumplir en la activación de algunas funciones propias de los relatos infantiles (véase el apartado siguiente), una identificación rigurosa de las mismas dota a la etapa posterior del estudio de un valor añadido en términos de consistencia.

Otro aspecto que debe tenerse en cuenta en la interacción entre texto e ilustraciones es la disposición de cada uno respecto al otro. Según Barone (2011), existen diversos rasgos anatómicos en los álbumes ilustrados, que determinan el modo en el que se percibirá y apreciará su contenido artístico. Entre estos rasgos se cuentan los márgenes, el contraste entre el color del texto y el de la imagen o, como se mencionaba anteriormente, la estructura empleada para incluir el texto y las ilustraciones a lo largo de las páginas del libro. En este sentido, existen múltiples variantes, con implicaciones distintas para la recepción del mensaje: situar la ilustración en el centro de la página o en un lado, colocarla junto al texto o debajo, incluir una o varias ilustraciones en una misma página, o crear ilustraciones que ocupen una página por completo (incluso dos), entre otras opciones.
En los ejemplos examinados en este trabajo, Frazee (2008) tiende a incluir el texto en primer lugar y la ilustración inmediatamente debajo. Sin embargo, esta no es una constante a lo largo del libro, y en el análisis puede verse que la ruptura de este patrón se emplea con intenciones muy concretas referentes al efecto que la información transmitida por el par texto-ilustración tendrá sobre el lector.

\section{Algunas funciones de la literatura infantil}

Según Anderson (2002), la literatura infantil viene a cumplir varias funciones, entre las que pueden destacarse la de incrementar el placer por la lectura, aprender a reconocer estructuras textuales, empatizar con los sentimientos y problemas de otras personas, aumentar el vocabulario, o reforzar los lazos entre el niño y el adulto, entre otras. En los álbumes ilustrados, los dibujos pueden ayudar a cumplir o reforzar algunas de estas funciones. El análisis del apartado siguiente busca demostrar, en última instancia, este punto a través del estudio de una selección de pasajes e ilustraciones de $A$ Couple of Boys Have the Best Week Ever.

Como se comentaba al principio de este artículo, en muchas ocasiones resulta complejo definir y delimitar el concepto de literatura infantil. Sin embargo, acerca de ello existe una categorización útil que abarca aspectos tan poliédricos como el tema, el tono o el contenido. Esta clasificación, propuesta por Wall (citado en Hunt, 2001, p.14), se organiza en torno al tipo y el número de destinatarios potenciales. Así, se establece una primera categoría en la que el autor escribiría directamente para un público infantil sin tener en cuenta a los adultos que puedan leer el texto en un momento dado. En segundo lugar, encontraríamos aquellas obras que van dirigidas a una audiencia doble; estos trabajos se orientan fundamentalmente a niños, pero toman también en consideración al adulto como potencial lector, incluyendo giros, bromas o referencias que al público de menor edad le puede resultar complicado entender. La última de las categorías propuestas por Wall (citado en Hunt, 2001, p. 14) es la de las obras destinadas a una audiencia dual, 
esto es, aquellas que, pese a tener al público infantil como receptor central, logran de algún modo despertar por igual el interés del adulto y del niño, y satisfacer análogamente a ambos colectivos.

Como se explicaba en la introducción, $A$ Couple of Boys Have the Best Week Ever es la historia de dos chicos que viven una semana de aventuras y diversión en pleno verano. Siguiendo los criterios de Wall, rasgos como la temática, el desarrollo de la historia o el vocabulario empleado llevan a descartar la orientación de la historia hacia una audiencia dual. Sin embargo, el tono irónico que la autora emplea en muchos casos, así como los chistes incluidos en ciertos pasajes, convierten el libro en un trabajo dirigido a una audiencia doble: niños en primera instancia, pero también aquellos adultos que podrán entender ciertos aspectos del libro, como el humor, con mayor facilidad.

Si la literatura infantil consta de tantas aristas como se comentaba en párrafos previos, parece complicado elaborar una relación breve de funciones principales. Es aquí donde considero importante recuperar la clasificación anterior, de cara a establecer el tipo de obra con el que estamos tratando a partir del público al que se orienta. El destinatario final de la obra debe ser un elemento clave a la hora de que el autor determinelasfunciones principales que su trabajo pretende cumplir.Ejemplificando a través de los extremos, un libro orientado únicamente a un público infantil tendrá objetivos distintos a los de una obra dirigida a una audiencia dual, donde el peso del adulto como figura receptora condicionará inevitablemente las funciones que esta pretende cumplir.

Asumiendo que el texto de estudio pueda catalogarse como dirigido a una audiencia doble y tomando en consideración el papel preponderante de la ilustración en el análisis, la siguiente relación de funciones propuesta por Anderson (2002) podría aplicarse a los márgenes de este estudio:

- Estimular el sentido de la estética del lector a través de las ilustraciones.
- Estimular y expandir su imaginación.

- Explorar los medios artísticos empleados en los dibujos.

- Dar vida a la historia.

- Prolongar el periodo de atención.

- Estimular el desarrollo cognitivo.

Dada la estrecha relación que cada uno de estos puntos guarda con los elementos visuales incluidos en los álbumes ilustrados, el último estadio del análisis se encarga de estudiar en qué medida el binomio textoilustración ayuda a reforzar (u opacar, en su caso) los seis propósitos listados anteriormente. Algunos de ellos permearán todos los ejemplos analizados en el apartado posterior; otros, sin embargo, tendrán una mayor o menor presencia, dependiendo de la dinámica que la autora haya decidido crear entre texto y dibujo, algo que el análisis traductológico del proceso de ilustración nos ayuda a determinar.

\section{Análisis}

Este apartado se centra en el estudio de tres pasajes del libro-álbum de Frazee (2008), A Couple of Boys Have the Best Week Ever, y sus correspondientes ilustraciones. Dicho análisis aúna los conceptos enunciados en el marco teórico con el fin de crear un modelo que permita ir alcanzando varios objetivos entrelazados: 1) identificar las decisiones tomadas por el ilustrador, mediante el uso de las estrategias y los procedimientos de traducción como principal herramienta; 2) establecer cómo estas decisiones determinan la interrelación entre texto e imagen en cada uno de los casos, y 3) describir el impacto de estas dinámicas sobre las principales funciones que cumple la literatura infantil.

Para llevar a cabo todo esto, en primer lugar, se presentan el pasaje y la ilustración en cuestión. A continuación, se incluye una tabla en la que se recoge información fundamental: tipo de estrategia de traducción aplicada por la autora, procedimientos de 


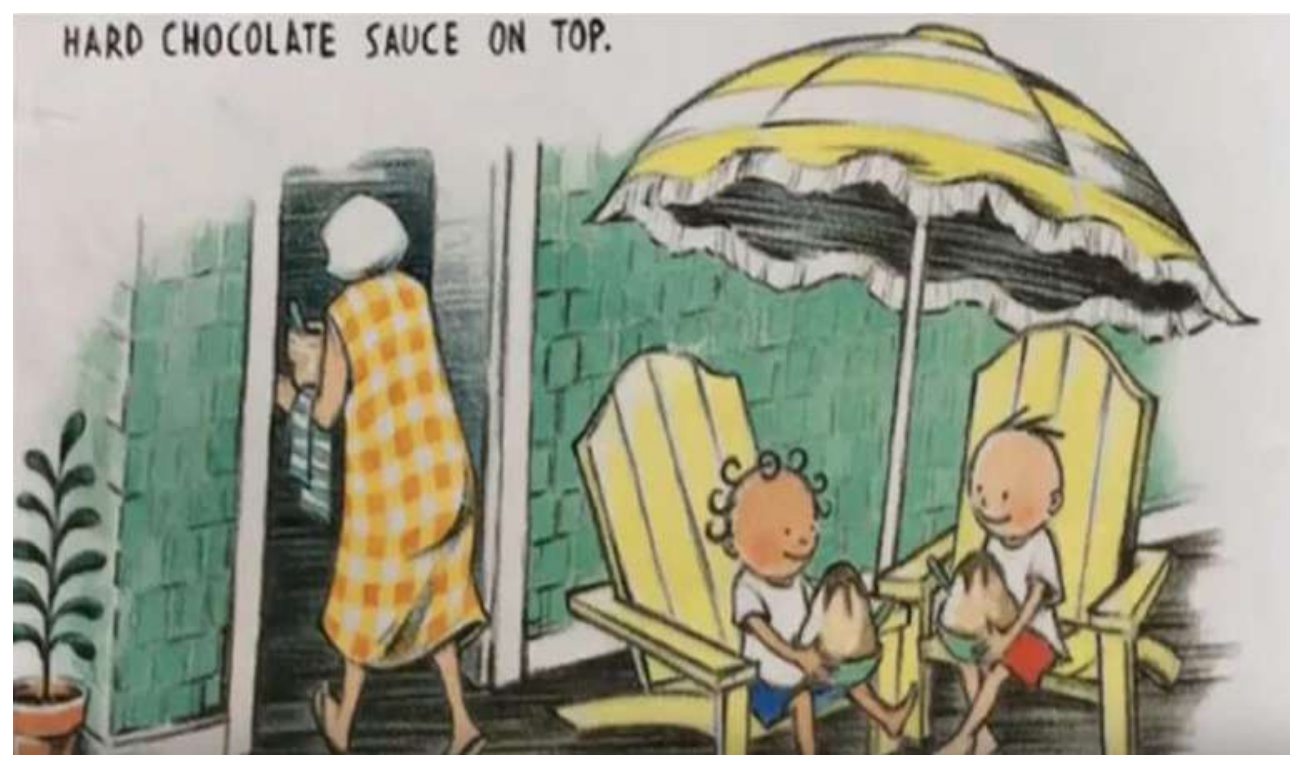

Figura 1 Elemento visual del ejemplo 1

Tabla 1 Análisis de ejemplo 1

\begin{tabular}{|c|c|c|c|}
\hline $\begin{array}{l}\text { Estrategia de } \\
\text { traducción }\end{array}$ & $\begin{array}{l}\text { Procedimientos de } \\
\text { traducción }\end{array}$ & Dinámica texto-ilustración & Funciones destacadas \\
\hline Traducción directa & $\begin{array}{l}\text { Traducción literal } \\
\text { Amplificación }\end{array}$ & De ampliación & $\begin{array}{l}\text { - Estimular el sentido de la es- } \\
\text { tética del lector a través de las } \\
\text { ilustraciones. } \\
\text { - } \quad \text { Explorar los medios artísticos } \\
\text { empleados en los dibujos. } \\
\text { - } \quad \text { Dar vida a la historia. } \\
\text { - Estimular el desarrollo cognitivo. }\end{array}$ \\
\hline
\end{tabular}

Fuente: Frazee (2008, p. 8).

traducción aplicados, dinámica texto-ilustración y funciones más destacadas del binomio formado por el elemento verbal y el visual. Estas tablas sirven como esquema para la discusión posterior, donde se explica con detalle cada uno de los puntos que aparecen en ellas.

\section{Ejemplo 1}

Elemento verbal: Pam gave James and Eamon coffee ice-cream icebergs with hard chocolate sauce on top (Frazee, 2008, p. 8). ${ }^{1}$

1 Al tratarse de un libro sin párrafos ni páginas numeradas, se aplica una numeración propia, contando como página 1 la posterior a los datos de publicación de la obra.
Elemento visual: en este primer ejemplo, la autora opta por enfocar el dibujo de la escena como una traducción directa, puesto que la imagen que incluye bajo el bloque de texto recoge los detalles descritos en el elemento verbal. En este sentido, varias piezas de información textual se traducen literalmente en la ilustración: la presencia de la abuela Pam, los dos niños tomando grandes bolas de helado o el sirope de chocolate que las recubre. En resumidas cuentas, a pesar de los cambios que analizamos en párrafos posteriores, el significado principal del texto se ve replicado en la ilustración de la autora (véase Figura 1).

Dicho esto, la autora también emplea el procedimiento de amplificación en varios puntos, 
relacionados fundamentalmente con el escenario en el que se mueven los personajes. Así, Frazee (2008) añade las sillas en las que los niños se sientan, una mesa o unas sombrillas, entre otros elementos. Pese a que ninguno de ellos aporta información que modifique o contradiga lo que se dice en el texto, sí agregan detalles relevantes para hacer que este cobre vida, ya que suman información contextual y ayudan al lector a descubrir el entorno en el que se mueven los protagonistas de la historia.

De este modo, las decisiones tomadas por Frazee, paralelas a varios procedimientos de traducción, generan una dinámica de ampliación entre el texto y la ilustración ya que, en este caso, la imagen añade una serie de detalles al texto que enriquecen el binomio. Como mencionábamos antes, el lector obtiene una reproducción fidedigna de lo escrito por la autora, al tiempo que suma nueva información que expande su conocimiento de la escena a través de la imagen. Este factor facilita que el lector tenga una perspectiva más comprehensiva de lo que les está ocurriendo a los personajes en ese momento. Plasmaciones tan detalladas como esta permiten fijar una imagen específica de lo ocurrido, lo cual tiene como contrapartida el bloqueo de la necesidad de inferencia o la limitación de las posibilidades imaginativas (Hunt, 2001). Por tanto, la relación de ampliación entre texto e ilustración establecida en este caso condiciona el potencial del binomio a otros niveles e impide explotar funciones propias de la literatura infantil como las que se analizan en ejemplos posteriores.

En lo que a estas funciones se refiere, este ejemplo sirve para desarrollar tres que permean los casos analizados y que, para evitar redundancias, se comentan fundamentalmente en este apartado. En primer lugar, la inclusión de imágenes en un texto es ya de por sí un mecanismo que, por su naturaleza visual y artística, estimula el sentido y la conciencia estética del lector. Asimismo, las ilustraciones presentes en un libro infantil permiten que, a partir de la propia curiosidad o con la guía de un adulto, el lector más joven pueda analizar los métodos empleados a la hora de dibujar la escena, los colores, la distribución y otros factores, estimulando así su sentido artístico. Por último, una de las funciones primordiales que cumplen las ilustraciones es la de ayudar a dar vida a la historia, poniendo cara y apariencia física a los personajes o aportando más detalles sobre el entorno que les rodea, como ocurre en este ejemplo. Esto, que en principio puede interpretarse como una ventaja y que es sin duda una de las principales consecuencias de ilustrar un libro, tiene como contrapunto la limitación de la imaginación del lector expuesta en el párrafo anterior, ya que el ilustrador determina, desde un primer momento, muchos aspectos que de otro modo podrían quedar al arbitrio de la inventiva de cada uno.

\section{Ejemplo 2}

Elemento verbal: And finally James did (p. 3) [...] with just a couple of his belongings (Frazee, 2008, pp. 3-4).

Elemento visual: en el segundo ejemplo, la autora opta por afrontar la ilustración como un proceso de traducción oblicua, ya que existen divergencias significativas entre la información que transmite el elemento verbal y la que obtenemos a través del elemento visual (véase Figura 2).

En este caso, la traducción literal se circunscribe a la presencia de James y su llegada a la casa de los abuelos de Eamon, mientras que el procedimiento central del ejemplo es la amplificación. Al contrario que en el primer caso, en el que las amplificaciones introducidas por Frazee no variaban la interacción entre el texto y la imagen, aquí vemos cómo el mismo procedimiento es la base sobre la que se genera una dinámica completamente distinta. De ahí que, como se mencionaba en el marco teórico, un mismo procedimiento pueda tener un impacto mayor o menor en función de la información que transmita y de su efecto en la dinámica texto-imagen.

Todo esto deriva, asimismo, en la presencia de un tercer procedimiento: la variación. En este caso, este fenómeno se produce por la transmutación, con base en amplificaciones que la autora 


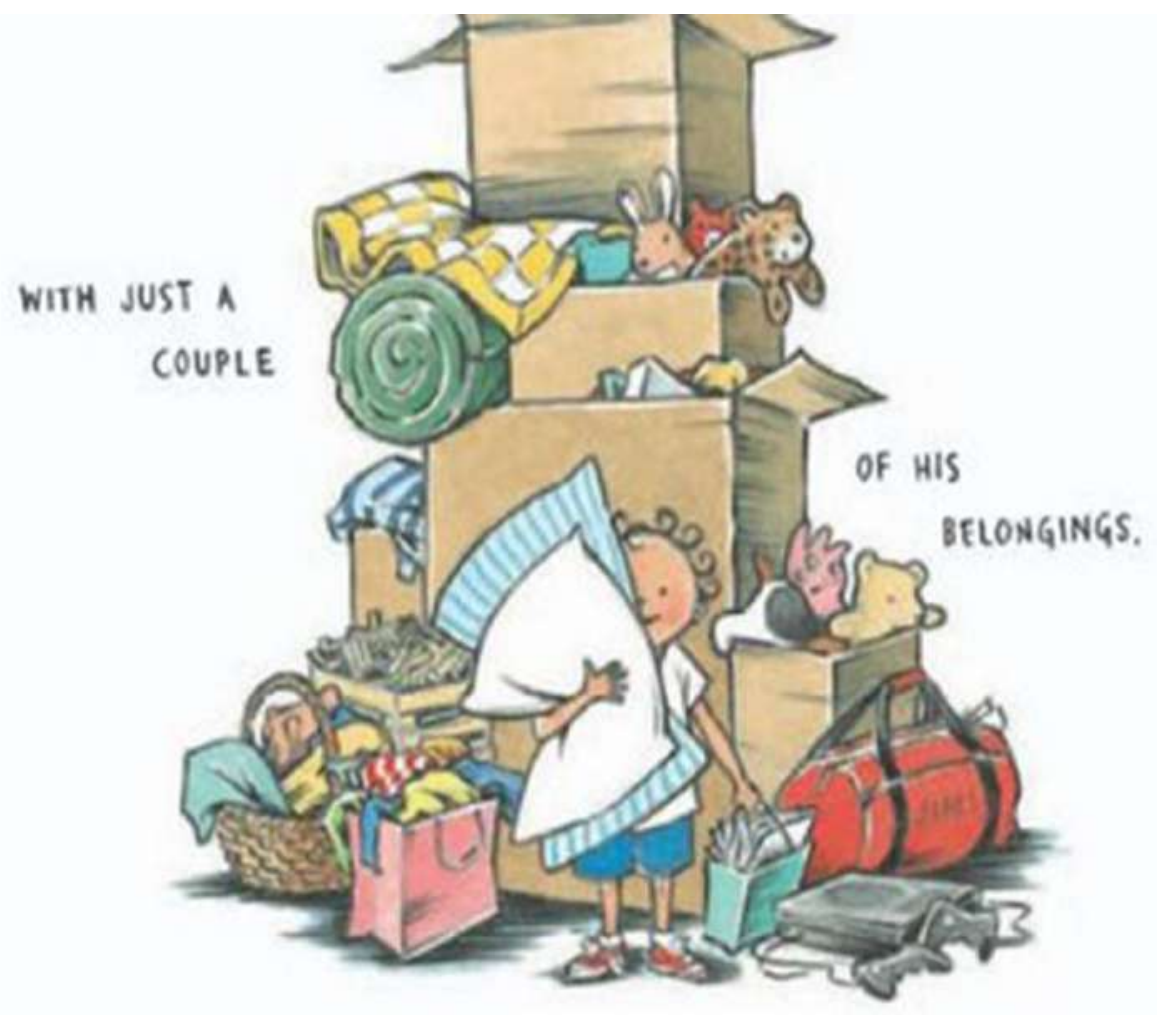

Figura 2 Elemento visual del ejemplo 2.

Tabla 2 Análisis de ejemplo 2

\begin{tabular}{|c|c|c|c|}
\hline $\begin{array}{l}\text { Estrategia de } \\
\text { traducción }\end{array}$ & $\begin{array}{l}\text { Procedimientos de } \\
\text { traducción }\end{array}$ & Dinámica texto-ilustración & Funciones destacadas \\
\hline Traducción oblicua & $\begin{array}{l}\text { Traducción literal } \\
\text { Amplificación } \\
\text { Variación }\end{array}$ & Contradictoria & $\begin{array}{l}\text { Estimular el sentido de la estética del } \\
\text { lector a través de las ilustraciones. } \\
\text { Estimular y expandir su imaginación } \\
\text { Explorar los medios artísticos empleados } \\
\text { en los dibujos. } \\
\text { Dar vida a la historia. } \\
\text { Prolongar el periodo de atención. } \\
\text { Estimular el desarrollo cognitivo. }\end{array}$ \\
\hline
\end{tabular}

Fuente: Frazee (2008, p. 3).

hace del elemento verbal y, más concretamente, del segmento "just a couple of". El cambio de información que aporta la imagen consigue que el tono del texto, completamente neutro y meramente descriptivo, cambie a un tono humorístico, al combinarse con el elemento visual.

Este último punto es un factor decisivo a la hora de determinar la dinámica que se establece entre el texto y la imagen en este caso. Teniendo en cuenta lo expuesto en el párrafo anterior, parece lógico deducir que nos encontramos ante un ejemplo evidente de contraposición: mientras que el elemento verbal describe las pocas pertenencias que acompañan a James, el elemento visual contradice esta información, al rodear al niño de gran cantidad de objetos. El principal efecto que logra esta oposición de mensajes es, como ya se mencionaba anteriormente, cómico. Pero en este binomio texto-ilustración intervienen aspectos adicionales que potencian este resultado. 
Así, otro elemento a destacar es el modo en el que texto e ilustración aparecen dispuestos. En contraposición a los ejemplos 1 y 3 , en los que un segmento textual completo y su "respuesta visual" aparecen al mismo tiempo, aquí la autora decide, a través de los puntos suspensivos, que el texto comience en una página y se cierre en la siguiente en conjunción con la ilustración. Esta estructura, unida al contexto de la escena (las dos páginas anteriores muestran a la familia sentada a la espera de James y el sonido del timbre a su llegada, lo que ya de por sí genera ciertas dosis de intriga en un lector que quiere ver qué se encontrarán los personajes al abrir la puerta), refuerza la sorpresa y el efecto humorístico que buscaba la autora, algo que podría haberse visto opacado de haber mantenido un patrón como el de los otros dos ejemplos analizados en este artículo.

Una de las principales funciones que se activa a partir de las explicaciones anteriores es el estímulo del desarrollo cognitivo, ya que el lector debe buscarle sentido a la contraposición de ideas entre imagen y texto. Al margen del tono humorístico que genera esta dinámica en cuestión, la oposición de información entre el elemento verbal y el visual transmite dos mensajes diferentes a la vez y a través de medios distintos. Esta ambigüedad supone un reto para el lector, que tiene que mediar entre ambos planos para poder deducir y entender lo que realmente está sucediendo (Nikolajeva y Scott, 2001). Asimismo, al no encontrar una explicación en el texto a la cantidad de objetos que James lleva a la casa de los abuelos de Eamon, el lector no puede sino imaginar las razones por las que esto ocurre. El hecho de no poder recurrir al texto para tratar de comprender un evento de la historia hace que el lector tenga que pensar y deducir el porqué de las cosas; esto supone un estímulo para su imaginación y un desafío más para sus capacidades de inferencia, factores que, según Serafini y Moses (2014), sirven también para espolear la curiosidad de los lectores y generar en ellos sentimientos de asombro.

Esta curiosidad y este asombro, creados a partir de las variaciones entre lo expresado por el elemento verbal y el visual, son factores que pueden ayudar, además, a mantener y concentrar la atención del lector. Frazee crea una contradicción que centra su interés y, conseguido este propósito, el papel de la imaginación en esta escena puede desplegarse en toda su amplitud, ya que la autora introduce numerosos estímulos sobre los cuales elucubrar, representados por los objetos que acompañan a James: ¿por qué lleva tantas cosas al campamento?, ¿qué papel cumplirá cada una?, ¿qué habrá dentro de cada caja y cada bolsa? Dicho con otras palabras, la dinámica texto-ilustración creada por Frazee consigue fijar la atención del lector en primer lugar para, posteriormente, fomentar su inventiva y reflexión a través de la disrupción entre la información del texto y la de la imagen.

\section{Ejemplo 3}

Elemento verbal: On their last night together, Bill, Pam, James, and Eamon had a pop corn party. James and Eamon soon discovered that a party with Bill and Pam could get pretty noisy (Frazee, 2008, p. 21).

Elemento visual: al igual que en el ejemplo anterior, nos encontramos ante un caso de traducción oblicua, en lo que a estrategia se refiere. Sin llegar al grado de distanciamiento apreciable en el segundo ejemplo de estudio, Frazee vuelve a optar por seguir una serie de procedimientos que alejan el elemento visual del verbal (véase Figura 3).

El primero de estos procedimientos que conviene destacar es la elisión que se produce, en tanto en cuanto la imagen no refleja el componente festivo (party) incluido en el texto. Viendo la ilustración de la autora, se entiende que la decisión es la de soslayar esa primera parte de la fiesta para centrarse en el final de esta, esto es, el segmento del texto que aparece reflejado en el elemento visual.

Si nos centramos en lo que la imagen nos ofrece, podemos identificar fácilmente otros dos procedimientos: traducción literal y amplificación. Dentro del primero, cabe englobar la inclusión de las figuras de Eamon y James, y la del bol de palomitas. Los principales casos de amplificación con los que 


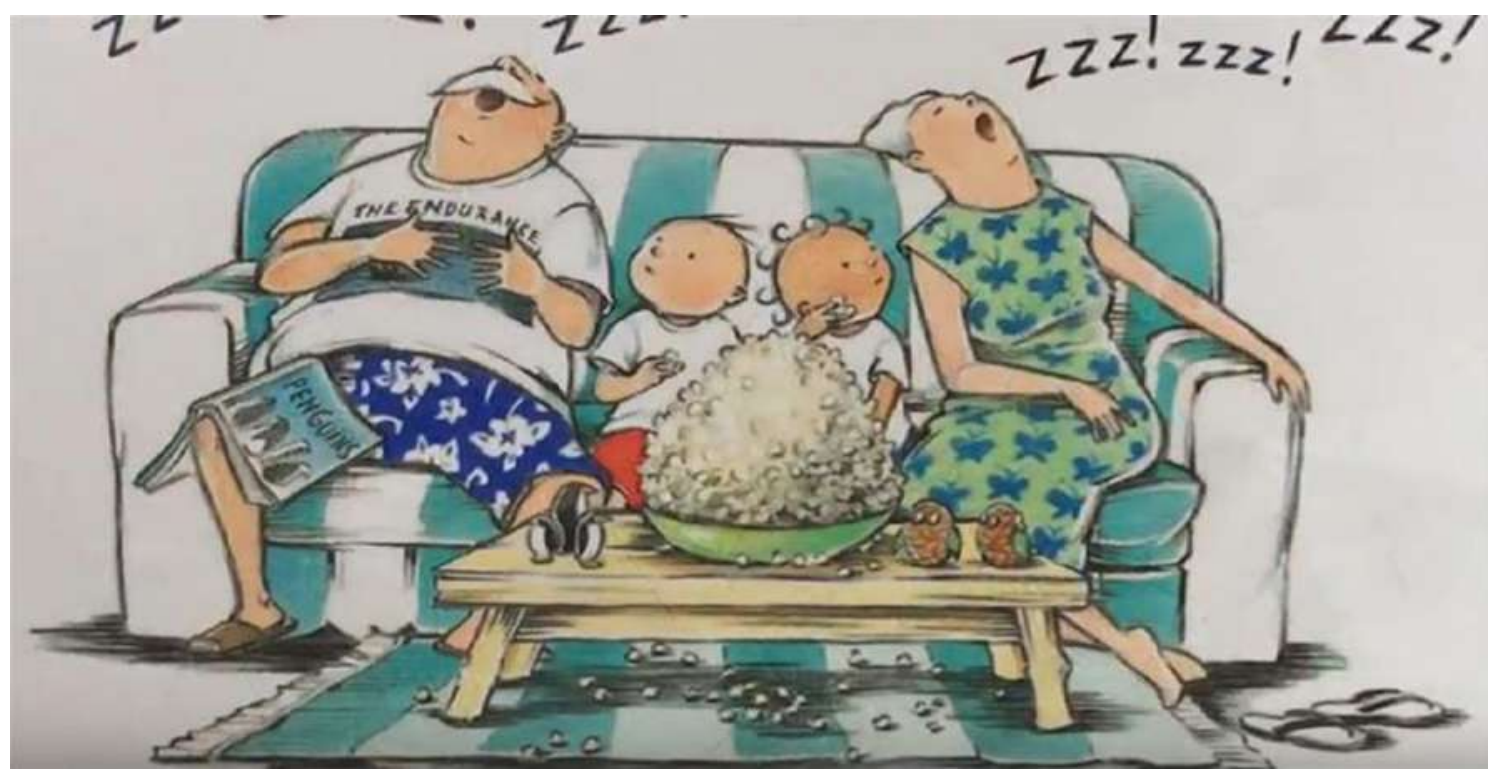

Figura 3 Elemento visual del ejemplo 3.

Tabla 3 Análisis de ejemplo 3

\begin{tabular}{|c|c|c|c|}
\hline $\begin{array}{l}\text { Estrategia de } \\
\text { traducción }\end{array}$ & $\begin{array}{l}\text { Procedimientos de } \\
\text { traducción }\end{array}$ & Dinámica texto-ilustración & Funciones destacadas \\
\hline Traducción oblicua & $\begin{array}{l}\text { Traducción literal } \\
\text { Amplificación } \\
\text { Elisión } \\
\text { Variación }\end{array}$ & Complementaria & 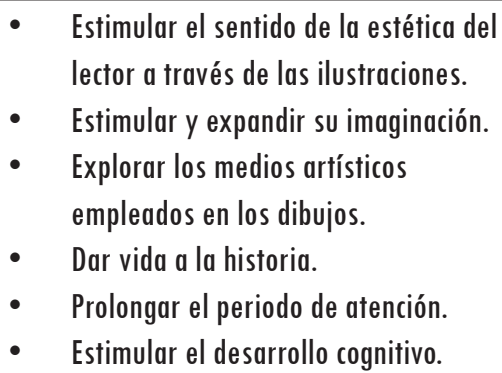 \\
\hline
\end{tabular}

Fuente: Frazee (2008, p. 21).

nos encontramos se relacionan con dos planos que, además, sirven para ejemplificar a la perfección los distintos grados de relevancia de elementos asociables a un mismo procedimiento. Por un lado, al igual que en el ejemplo 1, Frazee incluye objetos como la mesa o el sofá, que enmarcan la escena en un lugar concreto y aportan información nueva, aunque no especialmente relevante. Al igual que ocurría en el primer caso de estudio, estos elementos proporcionan detalles sobre el entorno en el que se desarrollan los acontecimientos y ayudan a que el texto cobre vida, pero por sí mismos no generan una distorsión entre el mensaje transmitido por el texto y el transmitido por la imagen.
Por otro lado, tenemos el caso de los abuelos de Eamon, Pam y Bill, a cuyo respecto Frazee decide añadir un rasgo adicional: el sueño. Así, incluye un retrato de ambos dormidos (algo que no se menciona en el texto de forma explícita) y dos largas series de zetas negras que, por onomatopéyica convencional, vienen a simbolizar los sonoros ronquidos de los personajes.

En este caso, la amplificación sí tiene un mayor impacto sobre el mensaje que transmite el binomio texto-ilustración, generando, al igual que en el ejemplo 2, un procedimiento de variación. Esta se produce fundamentalmente a partir del fragmento "a party with Bill and Pam could get pretty noisy". Al 
amplificar el contenido del texto con los elementos listados en el párrafo anterior, Frazee consigue cambiar la imagen habitual que el lector podría asociar a una fiesta ruidosa (gritos, música, gentío) con un giro humorístico centrado en el tremendo ruido que, se deduce, generan los ronquidos de los abuelos. De este modo, comprobamos que, en efecto, el segundo procedimiento de amplificación tiene un peso mayor que el primero, ya que deriva en un juego con la ambigüedad de las palabras y las presuposiciones del lector que acaba teniendo un peso significativo en la información que finalmente transmite el binomio texto-imagen.

El análisis de los procedimientos de traducción empleados por Frazee en esta ilustración nos permite establecer que la dinámica texto-imagen es complementaria. Al contrario que en el ejemplo 2 , en este no hay una contraposición de información entre el elemento verbal y el visual ("algunas pertenencias" vs. "cientos de cosas"), sino que se da un sentido particular al término "ruidoso", alejándolo del que tradicionalmente se asociaría a un ambiente festivo. Por tanto, no podemos hablar de contraposición de ideas, sino del añadido de información significativa en uno de los dos elementos, que obliga al lector a centrar su atención en la imagen, para comprender el significado real de lo que acontece en este fragmento.

Este punto entronca directamente con una de las funciones listadas en la tabla superior: la estimulación cognitiva. Al igual que ocurría en el segundo ejemplo, el lector tiene que contrastar la información que recibe desde el plano verbal y desde el plano visual, ya que entre ellas existen ciertas diferencias. La autora, en este caso, juega con la ambigüedad del término noisy, por lo que una vez analizadas ambas representaciones, el lector se verá en la necesidad de reflexionar para poder inferir que, en este pasaje del libro, una fiesta que se torna ruidosa no es sino una tarde de diversión que termina entre los ronquidos de Pam y Bill. Tanto el juego palabra-imagen propuesto por la autora como el efecto cómico final consiguen que se cumpla otra de las funciones mencionadas en la tabla: retener la atención del lector sobre lo que está leyendo/viendo.

Por último, siguiendo la línea del ejemplo 2, la dinámica creada por la autora sirve de nuevo para impulsar la imaginación de los lectores. En este tercer ejemplo, el elemento verbal habla de una fiesta de la que solo conocemos sus postrimerías. La elisión en la imagen de toda referencia a esa primera parte, unida a la representación visual de Bill y Pam, hará que, probablemente, el lector comprometido se pregunte en qué consistió esa fiesta de palomitas que terminó con los abuelos de Eamon durmiendo a pierna suelta.

\section{Conclusiones}

A lo largo de este artículo se ha demostrado que, tal y como se recogía en las hipótesis iniciales, existen varios paralelismos entre lo que supone traducir un texto y el acto de ilustrarlo. Estos paralelismos pueden verse, por ejemplo, en las analogías existentes entre ciertas decisiones tomadas a lo largo de ambos procesos.

Asumiendo estas premisas, las estrategias y los procedimientos de traducción permiten crear un andamiaje teórico aplicable al análisis de las transmutaciones texto-ilustración a partir del cual estudiar la labor llevada a cabo por el dibujante con respecto al pasaje de partida. Asimismo, al margen de su utilidad como instrumento de evaluación comparativa entre obra original y obra meta, este modelo facilita, en un segundo estadio, una identificación clara y precisa de la dinámica que genera el binomio texto-imagen, puesto que permite subrayar las piezas de información que ambos comparten, así como las omisiones, adiciones o modificaciones observables en su comparación.

Del mismo modo, este estudio viene a corroborar la fuerte ligazón existente entre las dinámicas texto-ilustración y ciertas funciones de la literatura infantil que pueden verse destacadas o ensombrecidas a través de ellas. En este sentido, el análisis realizado permite ver cómo, dependiendo de las 
estrategias y los procedimientos traductológicos que consciente o inconscientemente aplique el ilustrador, las dinámicas serán unas u otras y, en consecuencia, el binomio texto-imagen activará funciones distintas.

El análisis anterior prueba, asimismo, la complejidad que encierran las relaciones entre texto e ilustración y, por consiguiente, la interpretación de muchos álbumes ilustrados que, tras un primer vistazo, podrían catalogarse como simples o infantiles. Hunt (2001) cuestiona esta premisa, aludiendo que, con sus lógicas excepciones, este tipo de textos genera una serie de relaciones intra y extratextuales y plantea una cantidad de preguntas al lector a la hora de interpretarlas que los distancian considerablemente de una lectura sencilla. Estas y muchas otras variables aplicables a la interpretación de su contenido convierten los álbumes ilustrados de cualquier temática, apariencia visual u orientación en un amplio campo de análisis al que los estudios de traducción pueden aportar sus propios ángulos y enfoques.

\section{Referencias}

Anderson, N. A. (2002). Elementary children's literature. The basics for teachers and parents. Boston, MA: Allyn and Bacon.

Barone, D. M. (2011). Children's literature in the classroom: Engaginglifelong readers. Nueva York, NY: The Guilford Press.

Campos, H. (1992). Metalinguagem \& outras metas. São Paulo: Perspectiva.

Eco, U. (2008). Decir casi lo mismo. Barcelona: Lumen.

Frazee, M. (2008). A couple of boys have the best week ever. Orlando, FL: Harcourt, Inc.

Hunt, P. (2001). Children's literature. Oxford, Reino Unido: Blackwell Publishers.
Hurtado Albir, A. (2001). Traducción y traductología. introducción a la traductología. Madrid, España: Cátedra.

Jakobson, R. (1959/2000). On linguistic aspects of translation. En L. Venuti (Ed.), The translation studies reader (pp. 113-118). Nueva York, NY: Routledge.

Nikolajeva, M, y Scott, C. (2001). How picturebooks work. Nueva York, NY: Garland Publishing.

Pereira, N. M. (2007). Book illustration as intersemiotic translation: The case of Alice in Wonderland in Brazil. En D. Kenny y K. Ryou (Eds.), Across boundaries: International perspectives on translation studies (pp. 56-77). Newcastle, Reino Unido: Cambridge Scholars Publishing.

Pereira, N. M. (2008). Book illustration as (intersemiotic) translation: Pictures translating words. Meta, 53(1), 104-119. https://doi.org/10.7202/017977ar

Salmani, B., y Eghtesadi, Z. (2015). An intersemiotic approach towards translation of cover designs in retranslated classic novels. Theory and Practice in Language Studies, 5(6), 1185-1191. https://doi. org/10.17507/tpls.0506.09

Schwarcz, J. H. (1982). Ways of the Illustrator. Visual Communication in Children's Literature. Chicago, IL: American Library Association.

Serafini, F., y Moses, L. (2014). The roles of children's literature in the primary grades. The Reading Teacher, 67(6), 465-468. https://doi.org/10.1002/trtr.1236

Sipe, L. R. (1998). How picture books work: A semiotically framed theory of text-picture relationships. Children's Literature in Education, 29(2), 97-108. https://doi.org/10.1023/A:1022459009182

Sitzia, E. (2010). “Where is the prince?” Unlocking Doré’s illustration of Perrault's Cinderella. Relief, 4(2), 158-173. https://doi.org/10.18352/relief.543

Vinay, J. P., y Darbelnet, J. (1995). Comparative stylistics of French and English. A methodology for translation. Ámsterdam: John Benjamins. https://doi. org/10.1075/btl.11

How to reference this article: Echauri-Galván, Bruno (2020). Transmutación texto-ilustración: un modelo para el análisis de su efecto sobre las dinámicas y funciones de los libros infantiles. Íkala, Revista de Lenguaje y Cultura, 25(1), 173-187, http://doi.org/10.17533/udea.ikala.v25n01a08 